Japanese Journal of Physiology, 36, 1253-1260, 1986

\title{
Potentiation of Vasopressin Secretion by Footshocks in Rats
}

\author{
Tatsushi Onaka, Mitsuko Hamamura, and Kinji YagI \\ Department of Physiology, Jichi Medical School, \\ Kawachi-gun, Tochigi, 329-04 Japan
}

\begin{abstract}
Effects of footshocks (FS) on antidiuretic hormone (vasopressin, VP) in the plasma were studied in rats. Continuously applied FS of $60 \mathrm{~s}$ period with $5 \mathrm{~ms}$ pulses at $50 \mathrm{~Hz}$ frequency significantly increased VP as well as adrenocorticotrophic hormone (ACTH) in the plasma in a timeand shock intensity-dependent manner. Contrarily, the $50 \mathrm{~Hz}$ FS of $2 \mathrm{~s}$ period as repeated intermittently at every $15 \mathrm{~s}$ for over the period of 2,10 , and $30 \mathrm{~min}$ were much less effective for increasing plasma VP, whereas these intermittent FS increased plasma ACTH to an extremely high level. During the inter-shock intervals of $13 \mathrm{~s}$ between successive two shock periods rats exhibited a "freezing" behavior. Hypertonic saline or urethane injected I.P. immediately after termination of the intermittent FS significantly increased VP as well as ACTH in the plasma. These data clearly indicate that FS potentiate VP secretion and suggest the possibility that emotional stress may suppress the noxious stimuli-induced VP secretion.
\end{abstract}

Key words: antidiuretic hormone, vasopressin, footshock, ACTH, rat.

In an earlier bioassay study (MIRSKY et al., 1954), footshocks (FS) were shown to increase antidiuretic activity in the rat plasma. Recently, however, it has been demonstrated with radioimmunoassay (RIA) technique that FS of $0.5 \mathrm{~Hz}$ do not significantly elevate plasma level of antidiuretic hormone (vasopressin, VP) in rats (KNEPEL et al., 1982). On the other hand, our previous electrophysiological data have shown that VP-secreting neurosecretory cells in the rat supraoptic nucleus are excited by noxious stimuli (Hamamura et al., 1984; SHIBUKI and YAGI, 1986) and suggest the possibility that FS potentiate VP secretion by the pituitary. In addition ineffectiveness of FS with relatively low frequency for potentiation of VP secretion (KNEPEL et al., 1982) also suggest the possibility that the temporal pattern of footshock pulses may influence the effectiveness of FS. In an attempt to test these possibilities we have conducted a series of experiments with continuously or

Received for publication August 28, 1986 
intermittently applied high frequency FS in rats. Here we report that VP secretion is potentiated by continuously applied FS.

\section{METHODS}

Male rats of Wistar strain weighing between 266 and $349 \mathrm{~g}$ were used. The FS comprising $5 \mathrm{~ms}$ square wave pulses with a constant current intensity between 0 and $4 \mathrm{~mA}$ and repeated at $50 \mathrm{~Hz}$ were supplied by a stimulus isolator (Tektronix, 2620) either continuously for the period of $60 \mathrm{~s}$ or intermittently for the period of $2 \mathrm{~s}$ repeated at an interval of $15 \mathrm{~s}$. The FS were given to rats in a box of Lucite glass $(41.5 \mathrm{~cm}$ in depth and width, $60 \mathrm{~cm}$ in height) equipped with a grid floor. After termination of FS the trunk blood was collected by decapitation and the plasma sample was separated by centrifugation. The VP was further extracted from the plasma by acetone and diethyl ether and dried by a centrifuge evaporator (Yamato, RD-31). The amount of VP in the plasma was determined by RIA with a specific anti 8-arginine vasopressin antiserum (Mitsubishi Yuka cr UCB for data of Fig. 4), ${ }^{125} \mathrm{I}$-VP (NEN), and synthetic VP (Sigma, grade VI) as the reference. The sensitivity was $0.1 \mathrm{pg} /$ tube. The ACTH content in the plasma was determined with a RIA kit for human ACTH (Cis).

The data were statistically tested by Mann-Whitney $U$ test.

\section{RESULTS}

\section{Effects of continuously applied FS}

After continuously applied repetitive FS at $3 \mathrm{~mA}$ intensity for $60 \mathrm{~s}$ period, plasma ACTH was significantly increased as compared with that of unshocked control rats $(107 \pm 65 \mathrm{pg} / \mathrm{ml})$, reached the maximum $5 \mathrm{~min}$ after the stimulus onset and then decreased within $60 \mathrm{~min}$ to the level $1 \mathrm{~min}$ after the FS (Fig. 1). The VP level in the same plasma samples as those for ACTH was extremely high at $1 \mathrm{~min}$ after the stimulus onset, became lower afterwards and returned to the level of unshocked control rats within $10 \mathrm{~min}$ (Fig. 1).

Figure 2 shows the effects of shock intensity on plasma ACTH 5 min and VP $1 \mathrm{~min}$ after the stimulus onset. The amounts of these hormones increased as the shock intensity was increased. The minimum effective intensity to produce a significant increase in plasma level of these hormones was $1 \mathrm{~mA}$ for ACTH and $2 \mathrm{~mA}$ for VP. As seen in Fig. 2 plasma ACTH level reached the plateau at $4 \mathrm{~mA}$ but plasma VP level did not.

\section{Effects of intermittently applied FS}

Intermittent FS were given to rats for the period of 2,10 , or $30 \mathrm{~min}$ at either $0 \mathrm{~mA}$ intensity for the handling control or $3 \mathrm{~mA}$ intensity. As shown in Fig. 3 intermittent FS increased plasma ACTH significantly and extremely as compared with that of the time-matched paired control groups. After the intermittent FS of 10 

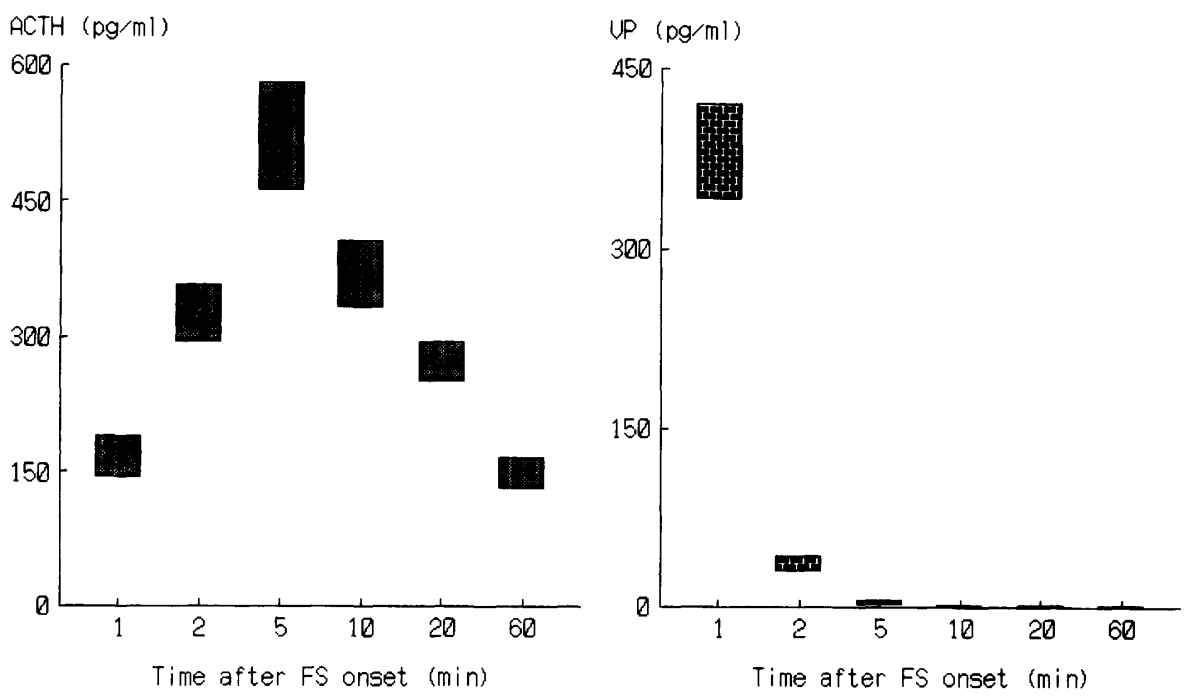

Fig. 1. Time courses of ACTH and VP in the plasma after continuously applied repetitive FS. Frequency and current intensity of stimulating $5 \mathrm{~ms}$ pulses were $50 \mathrm{~Hz}$ and $3 \mathrm{~mA}$. Each bar represents the range of mean \pm S.E.M. Number of rats in each group was 8 .
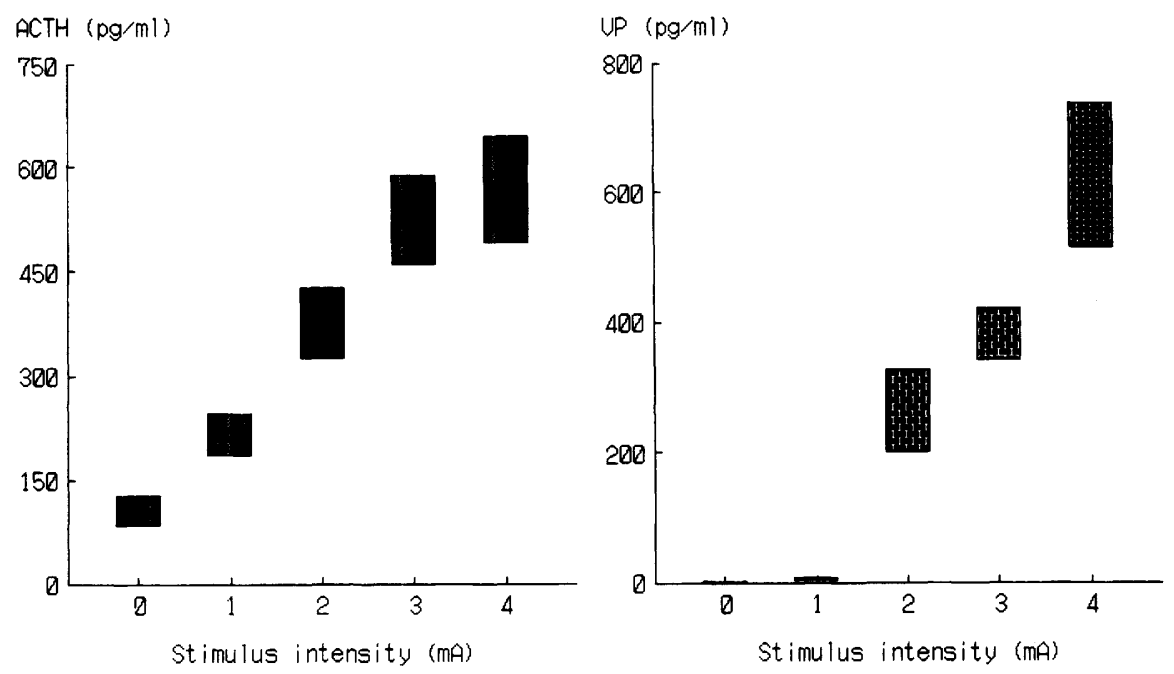

Fig. 2. Effects of footshock intensity on plasma ACTH 5 min after and plasma VP $1 \mathrm{~min}$ after the stimulus onset. Period of FS was $60 \mathrm{~s}$. Number of animals in the group of 1 and $4 \mathrm{~mA}$ intensity was 8 and that of the other group was 9 .

or $30 \mathrm{~min}$ period, plasma level of ACTH exceeded the level in the rats which received the continuous FS of 60 s period. Intermittent FS also increased plasma VP significantly but the magnitude of increase was much less than that in the rats which 

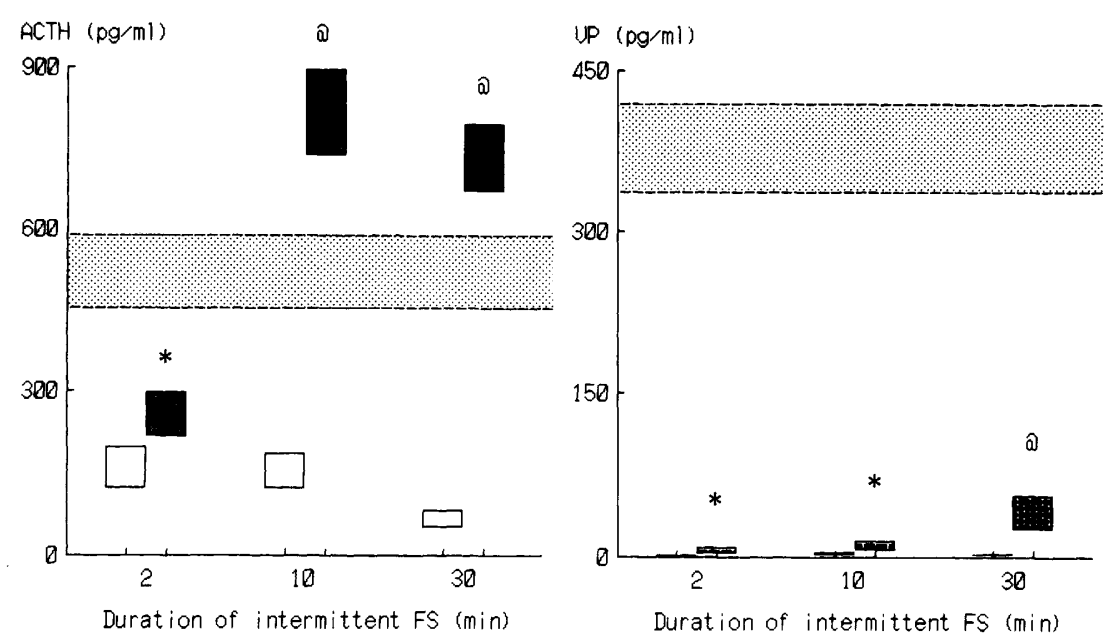

Fig. 3. Effects of intermittently applied $50 \mathrm{~Hz}$ FS on ACTH and VP in the plasma. Dark columns indicate plasma levels of hormoncs ir the blood obtained immediately after termination of the intermittent FS. Blank columns represent hormone levels in the paired control rats which were treated with $0 \mathrm{~mA}$ FS. Stippled area indicates either the range of plasma ACTH level in mean \pm S.E.M. $5 \mathrm{~min}$ after the onset of continuous FS or the range of plasma VP level 1 min after the FS onset. * and $@$ represent the significant level of $p<0.05$ and $p<0.002$ by MannWhitney $U$ test, respectively. Number of rats was 8 in the control and 4 in the experimental groups of 2 min duration. Number of rats in the other group was 7 .

received continuous FS of $60 \mathrm{~s}$ period (Fig. 3).

During the inter-shock intervals the rats exhibited and maintained an intense inhibition of general motor behavior which is known as "freezing" behavior.

To determine whether VP store in the pituitary had been exhausted after the intermittent FS we studied the potentiating effect of hypertonic saline or urethane I.P. injected immediately after termination of intermittent FS of $10 \mathrm{~min}$ period on plasma ACTH and VP in the blood collected 3 min afterwards. As shown in Fig. 4 VP but not ACTH in the plasma significantly increased after the hypertonic manipulation in the rats that had received intermittent FS of $10 \mathrm{~min}$ period. Urethane increased both ACTH and VP in the plasma to the extremely high levels in the rats that had received the intermittent FS (Fig. 4).

\section{DISCUSSION}

In the present experiments continuously applied high frequency repetitive FS were demonstrated to increase plasma VP in a time- and shock intensity-dependent manner. On the other hand, at least three possible factors have been known to potentiate VP secretion, namely an increase in body fluid osmolarity (VERNEY, 1947), acute hypovolemia or a blood pressure decrease (GINSBURG and BROWN, 

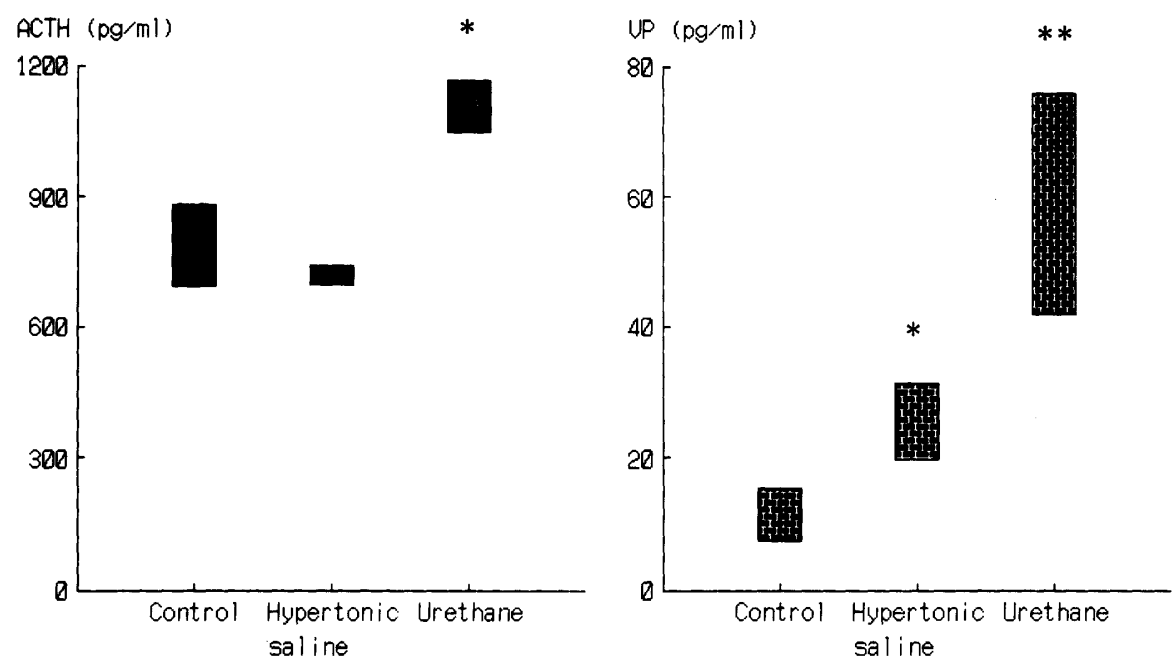

Fig. 4. Effects of hypertonic saline and urethane I.P. injected immediately after termination of intermittent FS of 10 min period on ACTH and VP in the plasma. Each column represents mean \pm S.E.M. of plasma ACTH or VP level in rats $3 \mathrm{~min}$ after termination of the intermittent FS. Control: uninjected control rats decapitated $3 \mathrm{~min}$ after termination of the intermittent FS. Hypertonic saline: rats injected I.P. with $1.54 \mathrm{M} \mathrm{NaCl}$ solution $(1.5 \%$ of body weight). Urethane: rats injected I.P. with urethane ( $1.75 \mathrm{~g}$ per $\mathrm{kg}$ body weight). Number of rats was 9 for the control and 8 for the other groups.

1956), and a vigorous exercise (BEARDWELl et al., 1975). The present results, therefore, might have been due secondarily to these factors rather than the direct neural influence of activated noxious afferents on the hypothalamic neuroendocrine system. However, it seems unlikely that FS might have significantly changed body fluid osmolarity under such an acute condition as $60 \mathrm{~s} \mathrm{FS}$. Activation of noxious afferents has been shown to excite supraoptic VP-secreting cells without mediation of a blood pressure decrease in anesthetized rats (HAmamura et al., 1984). It has been demonstrated that complete blocking of neuromuscular transmissions does not change the excitatory synaptic inputs to supraoptic VP-secreting cells after activation of cutaneous afferent nerve fibers in anesthetized rats (SHIBUKI and YAGI, 1986). Thus it is very likely that FS increase plasma VP at least in part as the consequence of activation of afferent neural pathways originating from nociceptors to hypothalamic VP-secreting cells. Furthermore, since both body fluid hyperosmolarity and hypovolemia simultaneously brought about by water deprivation in rats increase plasma VP up to $20 \mathrm{pg} / \mathrm{ml}$ (DuNN et al., 1973) and a vigorous exercise elevates plasma VP level to about $15 \mathrm{pg} / \mathrm{ml}$ in man (BEARDwELl et al., 1975), the maximal level of mean plasma VP of $628 \mathrm{pg} / \mathrm{ml}$ attained after the FS in the present experiments (Fig. 2) cannot be explained by those three factors having been reported to potentiate VP secretion. Thus it is reasonable to conclude that noxious 
stimuli such as FS potentiate VP secretion due to an activation of afferent neural pathways conveying signals from nociceptors to hypothalamic neurosecretory cells.

An earlier bioassay study has shown that noxious stimuli including FS increase plasma antidiuretic hormone activity in rats (MIRSKY et al., 1954). On the other hand a recent RIA study has demonstrated that FS with a relatively low frequency $(0.5 \mathrm{~Hz})$ do not increase plasma VP (KNEPEL et al., 1982). In the present experiments continuously applied high frequency FS $(50 \mathrm{~Hz})$ increased plasma VP to an extremely high level but intermittently applied FS were much less effective for increasing plasma VP than continuous FS were. Thus the differential responsiveness of the hypothalamo-neurohypophysial system to FS with different temporal pattern of stimulating pulses accounts for the apparent discrepancy among the reported data.

FS with a relatively low frequency $(0.5 \mathrm{~Hz})$ have been shown to be ineffective for stimulating VP secretion (KNEPEL et al., 1982). In the present experiments intermittent FS applied for the period between 2 and 30 min were consistently much less effective for increasing plasma VP than continuous FS were (Fig. 3). One possible explanation for the ineffectiveness of intermittent FS is that VP inactivation with a very rapid rate as reflected by the data in Fig. 1 might have lowered during inter-FS intervals the increased VP level after each intermittent FS. However, continuously applied FS with frequency as low as $0.5 \mathrm{~Hz}$ have been demonstrated to be ineffective for increasing plasma VP (KNEPEL et al., 1982). Another possible cause of ineffectiveness of intermittent FS may be an inhibitory action on primary afferent inputs, which has been shown to be produced by an activation of the "intrinsic analgesia system". (TERMAN et al., 1984). However, this mechanism does not explain satisfactorily the ineffectiveness of intermittent FS since plasma ACTH was increased to an extremely high level after the intermittent FS (Fig. 3). It may be also possible that the intermittent FS but not continuous FS might have exhausted a "readily releasable pool" of VP in the posterior pituitary (Thorn, 1966; DANiEl and Lederis, 1967; SACHS et al., 1967). However, this possibility is not likely because hypertonic saline or urethane administered I.P. to the rats immediately after termination of intermittent FS of $10 \mathrm{~min}$ period significantly increased plasma VP (Fig. 4). In the present experiments rats showed a "freezing" behavior during the inter-shock interval of intermittent FS. Since the "freezing" behavior is known as an indication of emotional stress of "fear" (DOYLE and YULE, 1959), the present data appear to suggest the possibility that emotional stress of "fear" suppresses the FS-induced VP secretion.

The present experiments have shown that noxious stimuli increase plasma VP. The physiological significance of a large amount of plasma VP after noxious stimuli is not known at present but has been hypothesized to facilitate memory consolidation (DE WIED, 1965), to produce analgesia (BERNTSON and BERSON, 1980), to facilitate circulatory homeostasis by water retention and vasoconstriction (SHIBUKI and YAGI, 1986), to potentiate ACTH secretion by the pituitary (RIVIER and VALE, 1983), and to facilitate homeostasis by increasing coagulant activity of the factor VIII 
in the plasma (Grant et al., 1985). These hypotheses remain to be tested.

We thank Dr. H. Ohno, Mitsubishi Yuka Co. for the generous gift of specific anti 8arginine vasopressin antiserum. This work was supported by Grants-in-Aid for Special Project Research from the Ministry of Education, Science and Culture of Japan.

\section{REFERENCES}

Beardwell, C. G., Geelen, G., Palmer, H. M., Roberts, D., and Salamonson, L. (1975) Radioimmunoassay of plasma vasopressin in physiological and pathological states in man. J. Endocrinol., 67: 189-202.

Berntson, G.. G. and Berson, B. S. (1980) Antinociceptive effects of intraventricular or systemic administration of vasopressin in the rat. Life Sci., 26: 455-459.

DANIEL, A. R. and LeDERIs, K. (1967) Release of neurohypophysial hormones in vitro. $J$. Physiol. (Lond.), 190: 171-187.

DE WIED, D. (1965) The influence of the posterior and intermediate lobe of the pituitary and pituitary peptides on the maintenance of a conditioned avoidance response in rats. Int. J. Neuropharmacol., 4: 157-167.

DoyLE, G. and Yule, E. P. (1959) Grooming activities and freezing behaviour in relation to emotionality in albino rats. Anim. Behav., 7: 18-22.

Dunn, F. L., Brennan, T. J., Nelson, A. E., and Robertson, G. L. (1973) The role of blood osmolality and volume in regulating vasopressin secretion in the rat. J. Clin. Invest., 52: 3212-3219.

GinsBurG, M. and Brown, L. M. (1956) Effect of anaesthetics and haemorrhage on the release of neurohypophysial antidiuretic hormone. Br. J. Pharmacol., 11: 236-244.

Grant, P. J., Davies, J. A., Tate, G. M., Boothby, M., and Prentice, C. R. M. (1985) Effects of physiological concentrations of vasopressin on haemostatic function in man. Clin. Sci., 69: 471-476.

Hamamura, M., Shibuki, K., and Yagi, K. (1984) Noxious inputs to supraoptic neurosecretory cells in the rat. Neurosci. Res., 2: 49-61.

KnePel, W., Nutto, D., and Hertting, G. (1982) Evidence for inhibition by $\beta$-endorphin of vasopressin release during foot shock-induced stress in the rat. Neuroendocrinology, 34: 353-356.

Mirsky, I. A., Stein, M., and Paulisch, G. (1954) The secretion of an antidiuretic substance into the circulation of rats exposed to noxious stimuli. Endocrinology, 54: 491-505.

Rivier, C. and Vale, W. (1983) Modulation of stress-induced ACTH release by corticotropin-releasing factor, catecholamines and vasopressin. Nature, 305: 325-327.

SACHS, H., Share, L., OsinchaK, J., and CarPI, A. (1967) Capacity of the neurohypophysis to release vasopressin. Endocrinology, 81: 755-770.

ShIBUKI, K. and YAGI, K. (1986) Synergistic activation of rat supraoptic neurosecretory neurons by noxious and hypovolemic stimuli. Exp. Brain Res., 62: 572-578.

Terman, G. W., Shavit, Y., Lewis, J. W., Cannon, J. T., and Liebeskind, J. C. (1984) Intrinsic mechanisms of pain inhibition: Activation by stress. Science, 226: $1270-1277$.

THORN, N. A. (1966) In vitro studies of the release mechanism for vasopressin in rats. Acta Endocrinol. (Kbh.), 53: 644-654. 
VERnEY, E. B. (1947) The antidiuretic hormone and the factors which determine its release. Proc. R. Soc. Lond. B, 135: 25-106. 